Article

\title{
Phytotoxicity Evaluation of Type B Trichothecenes Using a Chlamydomonas reinhardtii Model System
}

\section{Tadahiro Suzuki and Yumiko Iwahashi *}

Applied Microbiology Division, National Food Research Institute, 2-1-12 Kannon-dai, Tsukuba, Ibaraki 305-8642, Japan; E-Mail: suzut@affrc.go.jp

* Author to whom correspondence should be addressed; E-Mail: yumiko@affrc.go.jp; Tel.: +81-29-838-8103; Fax: +81-29-838-7996.

Received: 6 December 2013; in revised form: 14 January 2014 / Accepted: 15 January 2014 / Published: 28 January 2014

\begin{abstract}
Type B trichothecenes, which consist of deoxynivalenol (DON) and nivalenol (NIV) as the major end products, are produced by phytotoxic fungi, such as the Fusarium species, and pollute arable fields across the world. The DON toxicity has been investigated using various types of cell systems or animal bioassays. The evaluation of NIV toxicity, however, has been relatively restricted because of its lower level compared with DON. In this study, the Chlamydomonas reinhardtii testing system, which has been reported to have adequate NIV sensitivity, was reinvestigated under different mycotoxin concentrations and light conditions. The best concentration of DON and NIV, and their derivatives, for test conditions was found to be $25 \mathrm{ppm}\left(2.5 \times 10^{-2} \mathrm{mg} / \mathrm{mL}\right)$. In all light test conditions, DON, NIV, and fusarenon-X (FusX) indicated significant growth inhibition regardless of whether a light source existed, or under differential wavelength conditions. FusX growth was also influenced by changes in photon flux density. These results suggest that $C$. reinhardtii is an appropriate evaluation system for type B trichothecenes.
\end{abstract}

Keywords: type B trichothecene; Chlamydomonas reinhardtii; PPFD; LED; toxicity testing

\section{Introduction}

Trichothecene mycotoxins are produced by phytotoxic fungi such as the Fusarium species, which cause Fusarium head blight. These toxic fungi cause disease damage not only to plants that are used for food and feed crops but also to livestock and humans [1,2]. Trichothecene mycotoxins are 
categorized into several types and the type B trichothecene group includes deoxynivaelnol (DON), nivalenol (NIV), and their derivatives. They have a relatively low toxicity compared with type A trichothecenes, such as T-2 or HT-2 toxins, but the toxicity varies with differences in cell type or species. The general toxicity mechanism of trichothecene is $60 \mathrm{~S}$ ribosome binding, leading to translation inhibition [3]. This also causes inhibition of cellular regeneration and, consequently, trichothecene exposure can cause abdominalgia or diarrhea derived from cell inflammation. Because trichothecene mycotoxins pollute food crops and feed grains all over the world, several countries have been establishing restriction values against DON [4]. The restriction value is based on the results of evaluation studies. However, a comprehension of toxic characters is not always sufficient because of the differences in toxic characters between cell types or species. Recently, a number of researchers have suggested that a difference in import ability of the cellular transporter makes the difference in toxicity [5-8]. A Saccharomyces cerevisiae deletion mutant of the PDR5 gene, which encodes a pleiotropic drug resistance $\mathrm{ABC}$ transporter located on the plasma membrane, has increased DON sensitivity [8,9]. However, the deletion of the PDR5 gene does not result in high sensitivity to NIV [9], but Fusaronon-X (FusX, 4-acetyl-nivalenol), which is a NIV derivative, indicates a high toxicity [10]. Yeast evaluation systems cannot report NIV toxicity well. It is thought that NIV is not retained in yeast cells because of its structure. However, NIV indicates a relatively high toxicity to mammalian cells where it depends on the different cell types [11,12]. Mycotoxins also indicate toxicities to various plants. Abbas et al. [12] also reported the phytotoxicity of type B trichothecenes against Lemna pausicostata (duckweed), and the result of this study provides distinct phytotoxicity data. It has been indicated that duckweed is useful for toxicity testing [13], and recently, there are indications that duckweed research is developing. However, there is not yet enough genomic information, which is essential for understanding the mechanism of phytotoxicity. Meanwhile, former studies with barley leaf, Arabidopsis thaliana, or wheat roots have indicated a high toxicity of DON [14-16]. Toxicity evaluation with Arabidopsis seems to be a useful test system for trichothecene mycotoxins because several studies have suggested DON or T-2 toxin toxicities [17-19]. As for the study with wheat tissue segments, detailed phenotypic toxicity reactions to the mold invasion or the fusarium mycotoxin have been reported [20]. However, information about NIV toxicity is not enough even with plant test systems as plant systems have several problems. For example, the Arabidopsis system needs genetic modification to prepare the NIV sensitivity, and wheat genome knowledge is still developing. Hence, the Chlamydomonas reinhardtii model system is a useful candidate for the evaluation of the cell system except for mammalian cells.

Chlamydomonas reinhardtii is a monocellular green algae with chloroplasts, and it has been applied as an experimental material of photosynthesis, phototaxis or channelrhodopsin studies. Abundant genomic information has been gathered through these studies, and recently, the whole genome microarray of $C$. reinhardtii has been constructed [21]. Alexander et al. [22] evaluated trichothecene mycotoxins using the $C$. reinhardtii $\mathrm{CC} 125 \mathrm{mt}+$ wild-type strain. It can grow under heterotrophic conditions with low light intensity and it is managed as a model plant organism in photosynthesis studies. McCormick [23] implied that this model is also useful for NIV evaluation and its genome analysis is making progress [24]. Taken together with those reports, the usefulness of $C$. reinhardtii for Toxicity evaluation is continuously increasing. In this study, the $C$. reinhardtii system was used for evaluating type B trichothecenes, and the appropriate testing conditions were examined for expanding 
information on the mycotoxin toxicities against plant cells. On the basis that $C$. reinhardtii is the model organism for photosynthesis study, the relationship between mycotoxins and lighting conditions was investigated. This study will provide information on phytotoxicity, which is useful for the protection and regulation of various agricultural environments including plant factories with Light-emitting diode (LED) lighting.

\section{Results and Discussion}

\subsection{Mycotoxin Sensitivities to Trichothecenes and Lighting Conditions}

Chlamydomonas reinhardtii has a heterotrophic character as it grows in nutrient-rich conditions although it is an autotroph as it photosynthesizes. By its photosynthesis ability, even the high-salt medium (HSM minimum medium; [25]), which lacks nutrients for heterotrophic growth, is able to sustain cell cultivation under abundant lighting and aeration. However, in a pre-cultivation test without aeration, the HSM medium slowed growth considerably (data not shown). The 96-well plate used in this study was not suitable for aeration treatments but was appropriate for the mycotoxin exposure test. Therefore, the TAP medium was prepared instead of the HSM medium as this enables heterotrophic growth, and a fluorescent ceiling light with a photosynthetic photon flux density (PPFD) of $6 \mu \mathrm{mol} \mathrm{m} \mathrm{m}^{-2} \mathrm{~s}^{-1}$ was applied to give some light for cell growth. The growth curves of C. reinhardtii with $>1.0 \times 10^{-2} \mathrm{mg} / \mathrm{mL}(10 \mathrm{ppm})$ mycotoxin conditions indicated changes of toxicities, and at $2.5 \times 10^{-2} \mathrm{mg} / \mathrm{mL}(25 \mathrm{ppm}$ ), mycotoxins were readily identified (Figure 1a). Mycotoxin conditions of $<10$ ppm gave identical growth curves. The $3 A c D O N$ growth was similar to the control growth, but $15 \mathrm{AcDON}$ growth was less. The NIV and FusX growth curves indicated further low growth, and the DON growth indicated the lowest growth rate, which was derived from high toxicity. These results suggest that $25 \mathrm{ppm}$ of mycotoxin is the best test condition for the evaluation of type B trichothecenes. Alexander et al. [22] found that $80 \mu \mathrm{M}$ concentrations of mycotoxins was the best test condition and the $25 \mathrm{ppm}$ of trichothecene used in this study is close to $\sim 80 \mu \mathrm{M}$, thus agreeing with Alexander et al. despite using new trichothecene mycotoxins. NIV and FusX have not been previously compared using the $C$. reinhardtii testing system and NIV test condition studies that allow comparison with other trichothecenes have not been conducted. However, NIV has been reported to be the most toxic type B trichothecene compound [23]. This study also indicated that NIV is relatively toxic although DON had the most toxic character. These different results suggest that many parameters such as type of strain, static culture, media volume, lighting condition, and solvent (which are thought to be differential conditions) might have influenced the NIV toxicity result.

In the barley leaf model study with fluorescent and incandescent lights, the lighting condition in the DON exposure test induced the bleaching of chloroplasts [15]. In this study, light and dark conditions were compared because the effect of complete dark with trichothecene mycotoxin was unknown and may induce differences in C. reinhardtii growth. Both conditions were prepared with the TAP medium, and the light condition was conducted at $18 \mu \mathrm{mol} \mathrm{m} \mathrm{m}^{-2} \mathrm{~s}^{-1}$ of white LED and the dark condition was conducted at $0 \mu \mathrm{mol} \mathrm{m} \mathrm{m}^{-2} \mathrm{~s}^{-1}$. The growth curves obtained from both conditions showed the same pattern although the maximal values in the dark condition remained low (Figure 1b). The toxicities of DON and 3AcDON are nearly identical, and those of NIV and FusX also closely 
resembled each other. In addition, 15AcDON toxicity was between $3 \mathrm{AcDON}$ and FusX and above all, DON almost totally inhibited growth. This means that type B trichothecenes did not induce the characteristic inhibitory effect on $C$. reinhardtii photosynthesis and growth with the TAP medium. This was not just due to the heterotrophic conditions provided, because cell growth with light was not that much better than in dark conditions. Teramoto et al. [26] has reported that the mRNA level of Lhl4, which encodes a distant relative of light-harvesting Chl-a/b proteins in $C$. reinhardtii, was elevated according to the increase of light intensity with the TAP medium. This indicates that photosynthesis was activated by the increase of light intensity. The difference between light and dark conditions was only a difference in growth rate, which agrees with this research without mycotoxin condition. Taken together, it suggests that the light conditions like white light (including fluorescent light and LED) do not influence the sensitivity of $C$. reinhardtii to trichothecene toxicity.

Figure 1. Evaluation conditions for type $\mathrm{B}$ trichothecenes in the C. reinhardtii testing system. (a) Influence of mycotoxin concentration on $C$. reinhardtii growth where cells were incubated in a multi-well plate with constant fluorescent light; (b) Influence of lighting conditions on $C$. reinhardtii growth where the light condition was $18 \mu \mathrm{mol} \mathrm{m} \mathrm{m}^{-2}$ of white Light-emitting diode (LED). Dimethyl sulfoxide (DMSO) was the control. $\mathrm{A}_{655}=$ absorbance $655 \mathrm{~nm}$, Bars represent S.E. $(n=3) .0 .1-25 \mathrm{ppm}=1.0 \times 10^{-4}-2.5 \times 10^{-2} \mathrm{mg} / \mathrm{mL}$.

(a)
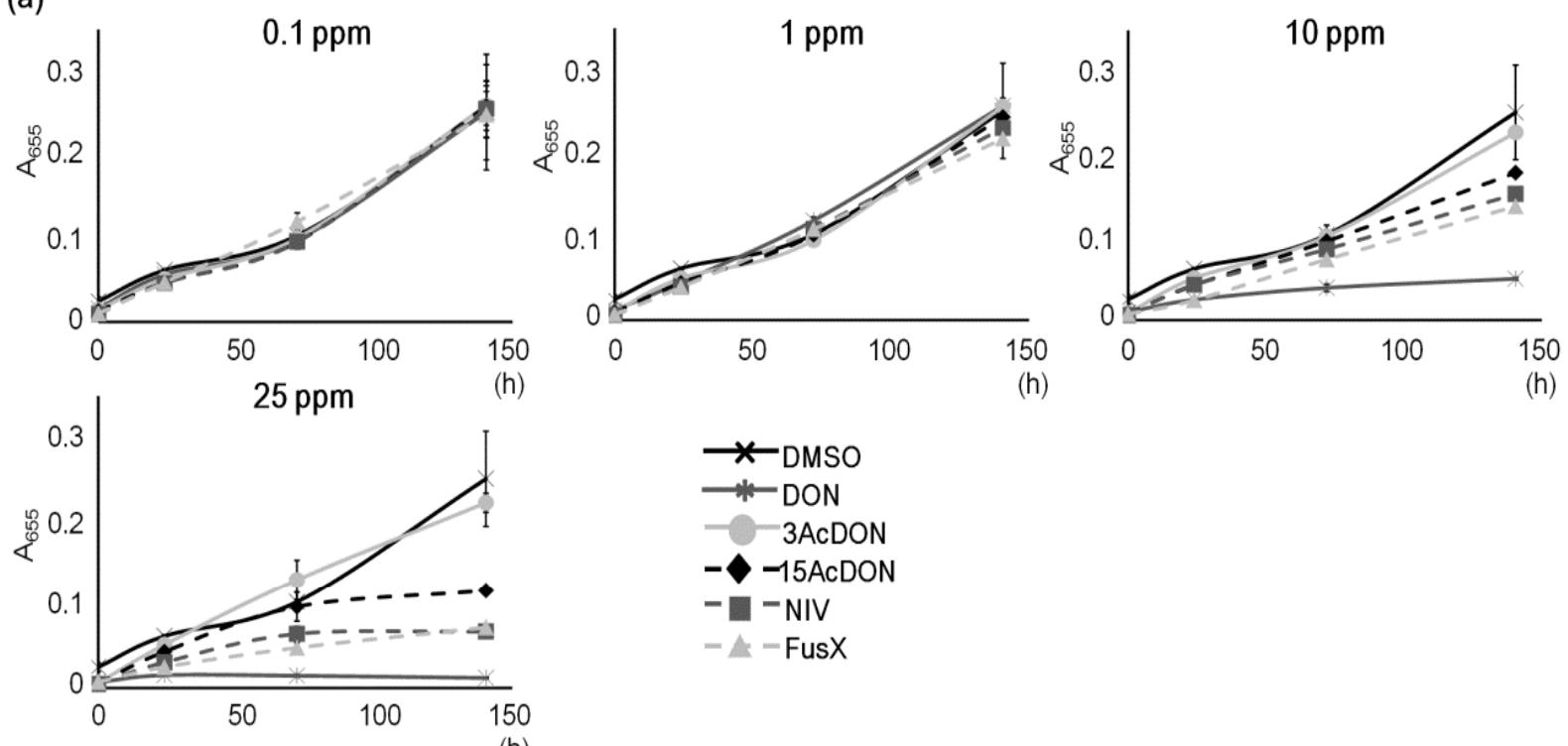

(h)

(b)
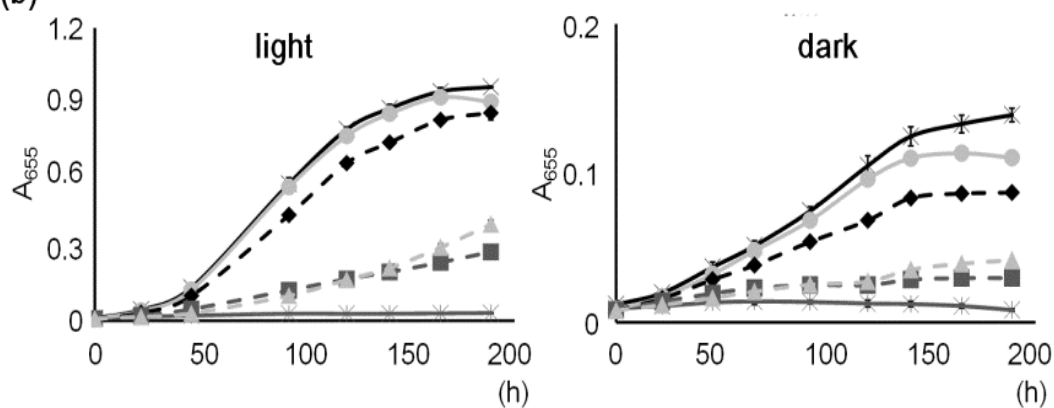
Figure 2. Spectral differences and sensitivities to mycotoxins. (a) LED spectral characters. Each graph is represented by the integration of irradiance. They only indicate spectral characters, not photosynthetic photon flux density (PPFD). Mix LED consists of the same components of red and blue LEDs. The maximal wavelength of each LED is indicated in the graph; (b) Growth test for comparing spectral characters. Lighting condition was $240 \mu \mathrm{mol} \mathrm{m}{ }^{-2} \mathrm{~s}^{-1}$ of each LED. DMSO was the control. $\mathrm{A}_{655}=$ absorbance $655 \mathrm{~nm}$, Bars represent S.E. $(n=3)$.
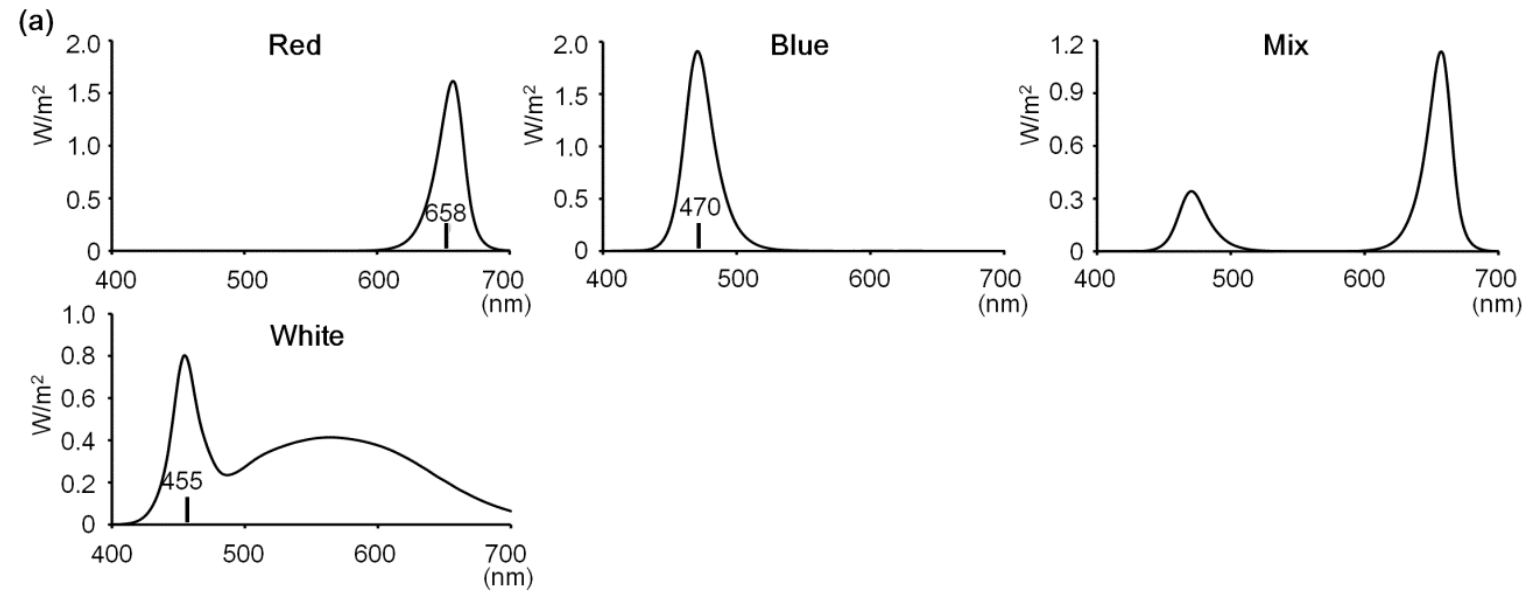

(b)
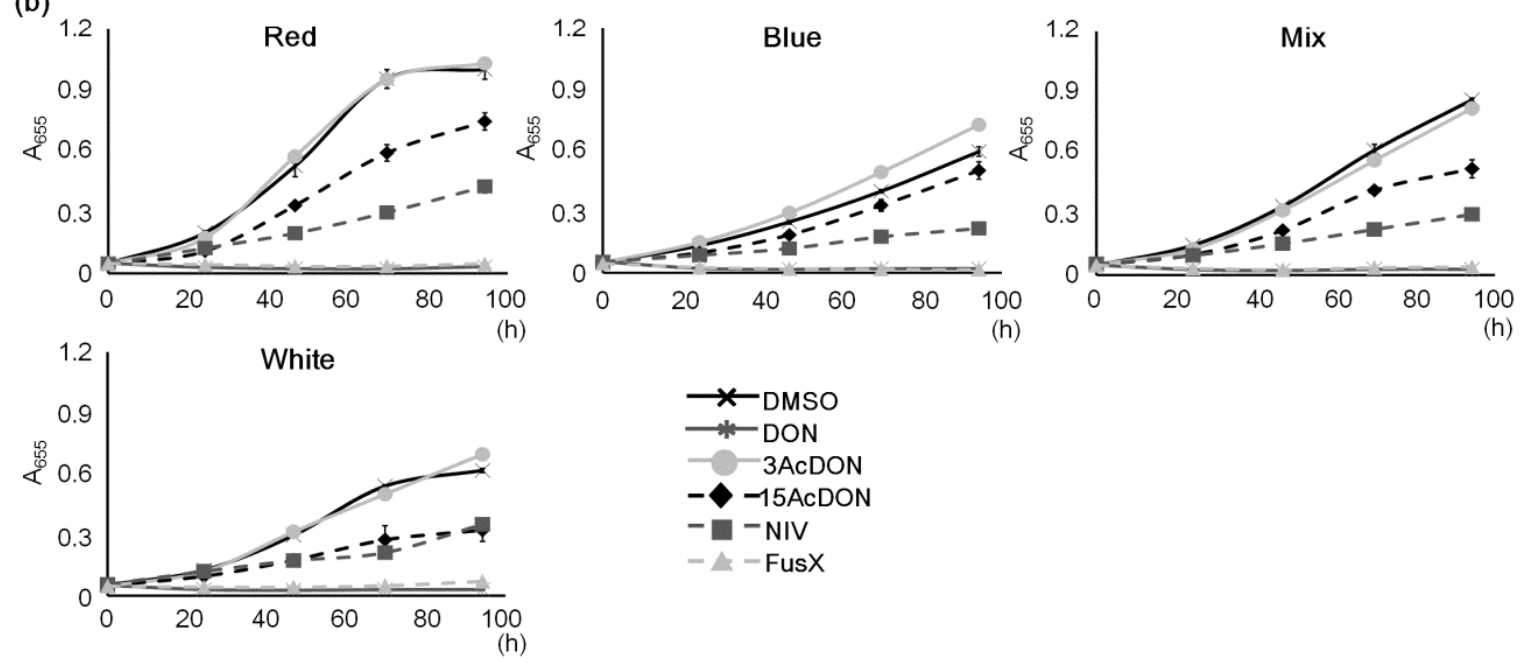

\subsection{Differences of Spectra}

Chlorophyll generally has two absorbance spectra; $\sim 450 \mathrm{~nm}$ and $\sim 660 \mathrm{~nm}$. Chlamydomonas reinhardtii also has the same character of absorbance spectra. The blue spectrum, which is mainly $450 \mathrm{~nm}$, is generally thought of as an important wavelength for $C$. reinhardtii growth because it regulates cell division and channel rhodopsin activation [27,28]. Additionally, it was reported that the blue spectrum induces more Lhl4 mRNA accumulation than green, red, far-red, or dark conditions but not white light conditions [26]. Katsuda et al. [29] applied 18-36 $\mu \mathrm{mol} \mathrm{m} \mathrm{m}^{-2} \mathrm{~s}^{-1}$ of blue LED in a photo-bioreactor to produce astaxanthin using a Haematococcus pluvialis system suggesting that blue LED is a useful testing condition. However, the most efficient growth of $C$. reinhardtii had been observed around the red spectra in a study that employed a chlorophyll synthesis mutant [30]. The red spectrum mainly consists of $660 \mathrm{~nm}$ and is also an important wavelength for photosynthesis. 
Differences in wavelength character influence various plant events as efficiencies of growth or photosynthesis. Various commercial LEDs can be obtained easily including many that carry appropriate bands for photosynthesis. Hence, in this study, blue, red, mix, and white LED conditions were tested (Figure 2a). A mix was prepared with a red-to-blue ratio of three to one. Red and blue LEDs were a bullet type, whereas the white LED, used as a general lighting control, was a bulb type. Each PPFD was set to $240 \mu \mathrm{mol} \mathrm{m} \mathrm{m}^{-2} \mathrm{~s}^{-1}$. All the growth data indicated the same trend to mycotoxin toxicities (Figure 2b). The 3AcDON condition did not indicate a characteristic difference compared with the control. Meanwhile, 15AcDON and DON indicated moderate or heavy toxicities, respectively. NIV indicated moderate changes, and FusX indicated high toxicities of the same degree as DON. Except under white LED conditions, NIV exposure indicated higher toxicities than that of $15 \mathrm{AcDON}$. The most characteristic difference was in cell growth rates between LED spectra conditions. The red LED condition was able to obtain the most characteristic differences because it was the best growth condition in this study. This growth trend corresponds with another study that found maximal chlorophyll content and cell volume in the red spectrum [30]. The result of this study suggests that red spectrum lighting is the most efficient evaluation condition because it gives rapid growth and characteristic growth rates. However, in blue and white LED conditions (both with a low contribution of red spectra), it seems that the toxicity level of $15 \mathrm{AcDON}$ was changing. Therefore, spectrum regulation for trichothecene mycotoxin evaluation should be employed to understand the relationship between mycotoxin toxicity and photosynthesis. The sun spectrum is constantly changing, and recent studies suggest that fungi have light-regulation systems [31,32]. Taken together, it is thought that this is a good approach to obtain fundamental information about the relationship between fungal invasion mechanisms and the day-length responses of plants.

\subsection{Photon Flux Conditions Affect FusX Sensitivity}

In this $C$. reinhardtii experimental system, a sufficient dose of PPF conditions enabled efficient growth and the identification of clear changes (Figure 2b). However, compared with lower dose conditions like in Figure 1, FusX did not grow as well as DON. It was not clear whether this problem was an accidental event, and therefore the influence of changes of PPFD was investigated. The red LED condition was selected because it results in the most efficient growth, and PPFDs were set up from 18.2 to $240 \mu \mathrm{mol} \mathrm{m} \mathrm{m}^{-2} \mathrm{~s}^{-1}$. The low PPFD was almost identical to the lighting condition of Figure $1 b$, the high PPFD value was almost identical to the lighting condition of Figure $2 b$, and the middle value was set to $87.6 \mu \mathrm{mol} \mathrm{m} \mathrm{m}^{-2} \mathrm{~s}^{-1}$. Except for FusX, almost all conditions did not give a significant change in growth curve (Figure 3). A low PPFD condition gave the most efficient growth in FusX and the middle and high PPFD conditions indicated extremely weak growth. Only $18.2 \mu \mathrm{mol} \mathrm{m}{ }^{-2}$ $\mathrm{s}^{-1}$ light condition indicated a statistical significance $(P<0.05)$. These results corresponded to the trends in Figures 1 and 2. Control conditions and 3AcDON conditions did not have any changes in growth curves. These results suggest that a change in PPFD only influences the sensitivity of C. reinhardtii to FusX. A higher toxicity of FusX than NIV has been reported from various cellular test conditions $[10,33]$. However, the difference in the plant pathogen toxicity mechanism between NIV and FusX is not clear. The lighting regulation will help to address this problem. 
Figure 3. Relationship between trichothecenes and photon flux. Photon flux density (PFD) of red LED is regulated at 18.2, 87.6, and $240 \mu \mathrm{mol} \mathrm{m} \mathrm{m}^{-2} \mathrm{~s}^{-1}$. DMSO was the control. $\mathrm{A}_{655}=$ absorbance $655 \mathrm{~nm}$, Bars represent S.E. $(n=3)$.
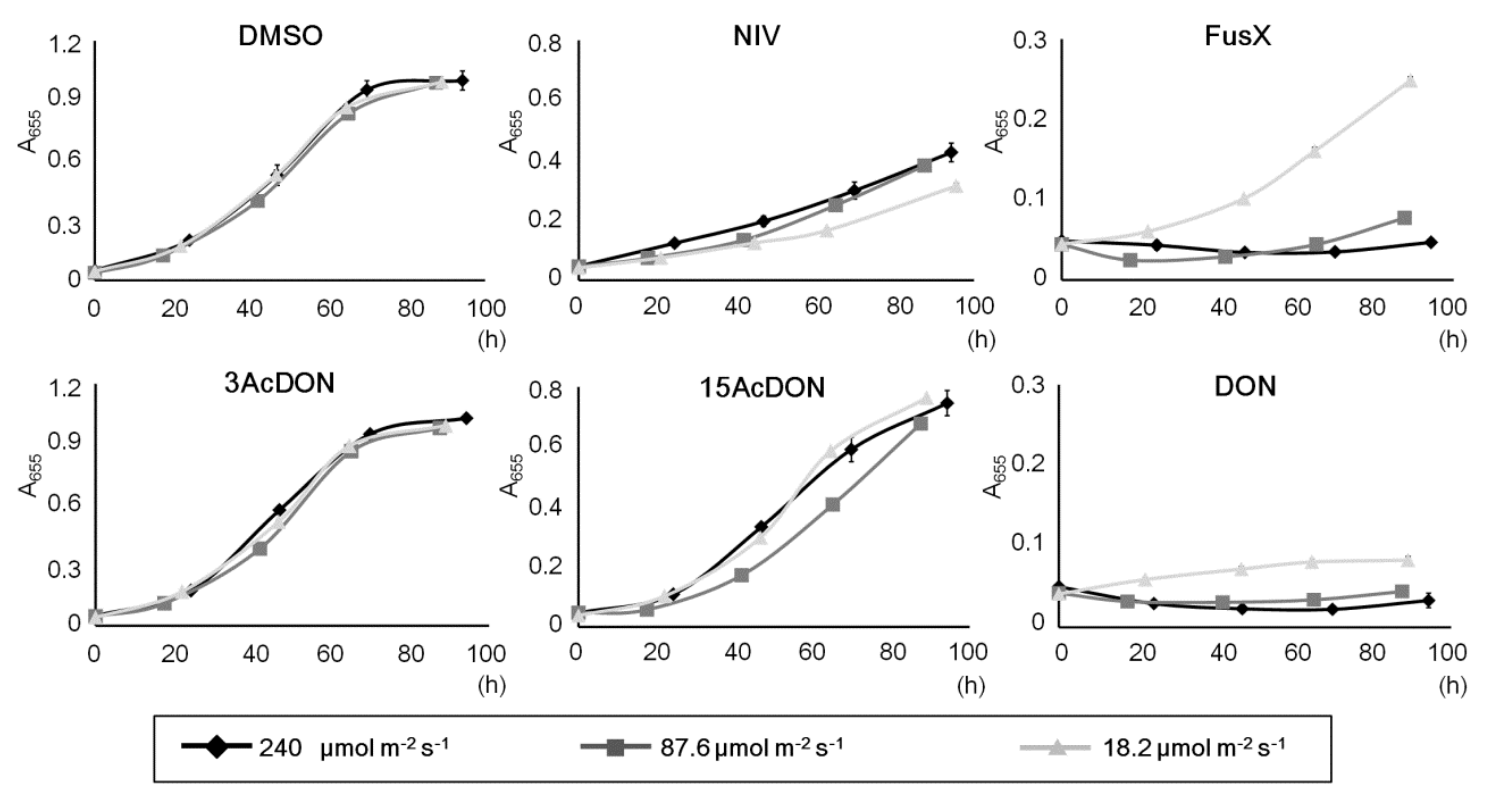

\section{Experimental Section}

\subsection{Strain, Medium, and Mycotoxins}

A colony of $C$. reinhardtii wild-type strain $137 \mathrm{C}$ was picked from slant culture on Tris-AcetatePhosphate (TAP) medium agar [34]. The TAP medium consists of three stock solutions. The TAP-salts solution was prepared from $40 \mathrm{~g}$ of $\mathrm{NH}_{4} \mathrm{Cl}, 10 \mathrm{~g}$ of $\mathrm{MgSO}_{4} .7 \mathrm{H}_{2} \mathrm{O}, 5 \mathrm{~g}$ of $\mathrm{CaCl}_{2} .2 \mathrm{H}_{2} \mathrm{O}, 242 \mathrm{~g}$ of Tris base, and $100 \mathrm{~mL}$ of acetic acid per $1 \mathrm{~L}$. The phosphate solution was prepared from $10.8 \mathrm{~g}$ of $\mathrm{K}_{2} \mathrm{HPO}_{4}$ and $5.6 \mathrm{~g}$ of $\mathrm{KH}_{2} \mathrm{PO}_{4}$. The trace elements solution was prepared from $5 \mathrm{~g}$ of $\mathrm{Na}_{2}$.EDTA, $2.2 \mathrm{~g}$ of $\mathrm{ZnSO}_{4} .7 \mathrm{H}_{2} \mathrm{O}, 1.14 \mathrm{~g}$ of $\mathrm{H}_{3} \mathrm{BO}_{3}, 0.51 \mathrm{~g}$ of $\mathrm{MnCl}_{2} .4 \mathrm{H}_{2} \mathrm{O}, 0.16 \mathrm{~g}$ of $\mathrm{CoCl}_{2} .6 \mathrm{H}_{2} \mathrm{O}, 0.16 \mathrm{~g}$ of $\mathrm{CuSO}_{4} .5 \mathrm{H}_{2} \mathrm{O}$, $0.11 \mathrm{~g}$ of $\left(\mathrm{NH}_{4}\right) 6 \mathrm{Mo}_{7} \mathrm{O}_{24} .4 \mathrm{H}_{2} \mathrm{O}, 0.5 \mathrm{~g}$ of $\mathrm{FeSO}_{4} .7 \mathrm{H}_{2} \mathrm{O}$, and $1.6 \mathrm{~g}$ of $\mathrm{KOH}$ per $1 \mathrm{~L}$. Chlamydomonas reinhardtii was pre-incubated at $100 \mathrm{rpm}$ rotation, at $25^{\circ} \mathrm{C}$, and constant lighting for more than 3 days. Each mycotoxin, DON, 3-acetyl-deoxynivalenol (3AcDON), 15-acetyl-deoxynivalenol (15AcDON), NIV, and fusaronon-X (FusX, 4acetyl-NIV) was dissolved in dimethyl sulfoxide (DMSO) to prepare a stock solution of $2000 \mathrm{ppm}$. A solution of only DMSO was used as a control.

\subsection{Light Source}

Light-emitting diode (LED) conditions were manually constructed on an LED platform (SPL-100-CC; REVOX, Kanagawa, Japan) with red $(660 \mathrm{~nm})$ and blue $(470 \mathrm{~nm})$ diodes, and photon flux density (PFD) was modulated by a pulse-width modulation dimmer controller. Mix LED consisted of both red and blue LEDs with a three-to-one ratio. Meanwhile, a white bulb-shaped LED was used as a general light control. Each spectrum of LED irradiation was measured by an illuminance spectrophotometer (CL-500A; Konica Minolta, Tokyo, Japan) as irradiances $\left(\mathrm{W} / \mathrm{m}^{2}\right)$. Total irradiances of spectra from 
400 to $700 \mathrm{~nm}$ were counted and the photosynthetic photon flux density (PPFD) of each spectrum condition was calculated with the following formula:

$\left[\operatorname{PPFD}\left(\mu \mathrm{mol} \mathrm{m} \mathrm{m}^{-2}\right)=\left(\left[\right.\right.\right.$ irradiance $\left.\left(\mathrm{W} / \mathrm{m}^{2}\right) \times \operatorname{spectrum}(\mathrm{m}) \times 10^{-9}\right] /[$ Planck's constant $(6.626 \times$ $\left.10^{-34} ; \mathrm{J} . \mathrm{s}\right) \times$ Speed of light $\left(2.998 \times 10^{8} ; \mathrm{m} / \mathrm{s}\right) \times$ Avogadro constant $\left.\left.\left.\left(6.022 \times 10^{23} ; \mathrm{mol}^{-1}\right)\right]\right) \times 10^{6}\right]$

\subsection{Growth Test Condition}

TAP-based cell cultures were incubated with $1.0 \times 10^{-4}-2.5 \times 10^{-2} \mathrm{mg} / \mathrm{mL}(0.1-25 \mathrm{ppm})$ of the mycotoxins, or DMSO only as a control. Each total culture volume was adjusted to $200 \mu \mathrm{L}$, and DMSO, which is a solvent, was adjusted to the low concentration value because an increase in DMSO influences $C$. reinhardtii growth negatively [35]. Sorted cultures in 96-well flat bottom micro plates were placed under the LED lighting at $25{ }^{\circ} \mathrm{C}$. Culture plates were transferred into a plate reader (iMark; Bio-Rad, Hercules, CA, USA) once a day, and then absorbance at $655 \mathrm{~nm}$ was measured. Each test was conducted with triplet samples. Growth curves of the FusX exposure test were analyzed using the T-test, and $240 \mu \mathrm{mol} \mathrm{m} \mathrm{m}^{-2} \mathrm{~s}^{-1}$ lighting condition was defined as a control.

\section{Conclusions}

In this study, trichothecene evaluation conditions were determined using $C$. reinhardtii as a model system. This system is advantageous for the evaluation of NIV toxicity where yeast or higher plants cannot be used as a test system. When evaluating mycotoxin toxicities, the effects of lighting conditions have not really been investigated. The results of this study suggested that differences in the light spectrum or incident density influence several trichothecenes. Recently, the whole genome microarray of $C$. reinhardtii has been constructed [21], and therefore the $C$. reinhardtii system has the ability to bring gene expression data derived from the differences of sensitivity against type B trichothecenes. Because the finding derived from gene expression analysis can be integrated with various cell systems, this system will become more useful, especially for investigating the relationship between the photosynthetic pathway and mycotoxins.

\section{Acknowledgments}

We are deeply grateful to M. Tsuzuki and Associate S. Fujiwara (Department of Applied Life Science, Tokyo University of Pharmacy and Life Science, Tokyo, Japan) for providing the C. reinhardtii strain and technical information.

\section{Conflicts of Interest}

The authors declare no conflict of interest.

\section{References}

1. Chowdhury, S.R.; Smith, T.K.; Boermans, H.J.; Sefton, A.E.; Downey, R.; Woodward, B. Effects of feeding blends of grains naturally contaminated with Fusarium mycotoxins on performance, metabolism, hematology, and immunocompetence of ducklings. Poult. Sci. 2005, 84, 1179-1185. 
2. Pestka, J.J. Deoxynivalenol: Toxicity, mechanisms and animal health risks. Anim. Feed Sci. Technol. 2007, 137, 283-298.

3. Pestka, J.J. Deoxynivalenol: Mechanisms of action, human exposure, and toxicological relevance. Arch. Toxicol. 2010, 84, 663-679.

4. FAO Food Nutrition Paper. Worldwide Regulations for Mycotoxins in Food and Feed in 2003; Food Quality and Standards Service: Rome, Italy, 2004.

5. Alexander, N.J.; McCormick, S.P.; Hohn, T.M. TRI12, a trichothecene efflux pump from Fusarium sporotrichioides: Gene isolation and expression in yeast. Mol. Gen. Genet. 1999, 261, 977-984.

6. Mitterbauer, R.; Adam, G. Saccharomyces cerevisae and Arabidopsis thaliana: Useful model systems for the identification of molecular mechanisms involved in resistance of plants to toxins. Eur. J. Plant Pathol. 2002, 108, 699-703.

7. Ohsato, S.; Ochiai-Fukuda, T.; Nishiuchi, T.; Takahashi-Ando, N.; Koizumi, S.; Hamamoto, H.; Kudo, T.; Yamaguchi, I.; Kimura, M. Transgenic rice plants expressing trichothecene 3-Oacetyltransferase show resistance to the Fusarium phytotoxin deoxynivalenol. Plant Cell Rep. 2007, 26, 531-538.

8. Abolmaali, S.; Mitterbauer, R.; Spadiut, O.; Peruci, M.; Weindorfer, H.; Lucyshyn, D.; Ellersdorfer, G.; Lemmens, M.; Moll, W.D.; Adam, G. Engineered bakers yeast as a sensitive bioassay indicator organism for the trichothecene toxin deoxynivalenol. J. Microbiol. Methods 2008, 72, 306-312.

9. Suzuki, T.; Sirisattha, S.; Mori, K. Iwahashi, Y. Mycotoxin toxicity in Saccharomyces cerevisiae differs depending on gene mutations. Food Sci. Technol. Res. 2009, 6, 453-458.

10. Suzuki, T.; Iwahashi, Y. Comprehensive gene expression analysis of type B trichothecenes. J. Agric. Food Chem. 2012, 60, 9519-9527.

11. Alassane-Kpembi, I.; Kolf-Clauw, M.; Gauthier, T.; Abrami, R.; Abiola, F. A.; Oswald, I.P.; Puel, O. New insights into mycotoxin mixtures: The toxicity of low doses of Type B trichothecenes on intestinal epithelial cells is synergistic. Toxicol. Appl. Pharmacol. 2013, 272, 191-198.

12. Abbas, H.K.; Yoshizawa, T.; Shier, W.T. Cytotoxicity and phytotoxicity of trichothecene mycotoxins produced by Fusarium spp. Toxicon 2013, 74, 68-75.

13. Wang, W. Literature review on duckweed toxicity testing. Environ. Res. 1990, 52, 7-22.

14. Desjardins, A.E.; McCormick, S.P.; Appell, M. Structure-activity relationships of trichothecene toxins in an Arabidopsis thaliana leaf assay. J. Agric. Food Chem. 2007, 55, 6487-6492.

15. Bushnell, W.R.; Perkins-Veazie, P.; Russo, V.M.; Collins, J.; Seeland, T.M. Effects of deoxynivalenol on content of chloroplast pigments in barley leaf tissues. Phytopathology 2010, 100, 33-41.

16. Ito, M.; Sato, I.; Ishizaka, M.; Yoshida, S.; Koitabashi, M.; Tsushima, S. Bacterial cytochrome P450 system catabolizing the Fusarium toxin deoxynivalenol. Appl. Environ. Microbiol. 2013, 79, 1619-1628.

17. Urban, M.; Daniels, S.; Mott, E.; Hammond-Kosack, K. Arabidopsis is susceptible to the cereal ear blight fungal pathogens Fusarium graminearum and Fusarium culmorum. Plant J. 2002, 32, 961-973. 
18. Nishiuchi, T.; Masuda, D.; Nakashita, H.; Ichimura, K.; Shinozaki, K.; Yoshida, S.; Kimura, M.; Yamaguchi, I.; Yamaguchi, K. Fusarium phytotoxin trichothecenes have an elicitor-like activity in Arabidopsis thaliana, but the activity differed significantly among their molecular species. Mol. Plant Microbe Interact. 2006, 19, 512-520.

19. Masuda, D.; Ishida, M.; Yamaguchi, K.; Yamaguchi, I.; Kimura, M.; Nishiuchi, T. Phytotoxic effects of trichothecenes on the growth and morphology of Arabidopsis thaliana. J. Exp. Bot. 2007, 58, 1617-1626.

20. Peraldi, A.; Beccari, G.; Steed, A.; Nicholson, P. Brachypodium distachyon: A new pathosystem to study Fusarium head blight and other Fusarium diseases of wheat. BMC Plant Biol. 2011, 11,100 .

21. Toepel, J.; Albaum, S.P.; Arvidsson, S.; Goesmann, A.; la Russa, M.; Rogge, K.; Kruse, O. Construction and evaluation of a whole genome microarray of Chlamydomonas reinhardtii. BMC Genomics 2011, 12, 579.

22. Alexander, N.J.; McCormick, S.P.; Ziegenhorn, S.L. Phytotoxicity of selected trichothecenes using Chlamydomonas reinhardtii as a model systemt. Nat. Toxins 1999, 7, 265-269.

23. McCormick, S.P. Phytotoxicity of Trichothecenes; American Chemical Society: Washington, DC, USA, 2009; Volume 1031, pp. 143-155.

24. Merchant, S.S.; Prochnik, S.E.; Vallon, O.; Harris, E.H.; Karpowicz, S.J.; Witman, G.B.; Terry, A.; Salamov, A.; Fritz-Laylin, L.K.; Maréchal-Drouard, L.; et al. The Chlamydomonas genome reveals the evolution of key animal and plant functions. Science 2007, 318, 245-250.

25. Sueoka, N. Mitotic replication of deoxyribonucleic acid in Chlamydomonas reinhardi. Proc. Natl. Acad. Sci. USA 1960, 46, 83-91.

26. Teramoto, H.; Ishii, A.; Kimura, Y.; Hasegawa, K.; Nakazawa, S.; Nakamura, T.; Higashi, S.; Watanabe, M.; Ono, T.A. Action spectrum for expression of the high intensity light-inducible Lhc-like gene Lhl4 in the green alga Chlamydomonas reinhardtii. Plant Cell Physiol. 2006, 47, 419-425.

27. Münzner, P.; Voigt, J. Blue light regulation of cell division in Chlamydomonas reinhardtii. Plant Physiol. 1992, 99, 1370-1375.

28. Nagel, G.; Szellas, T.; Huhn, W.; Kateriya, S.; Adeishvili, N.; Berthold, P.; Ollig, D.; Hegemann, P.; Bamberg, E. Channelrhodopsin-2, a directly light-gated cation-selective membrane channel. Proc. Natl. Acad. Sci. USA 2003, 100, 13940-13945.

29. Katsuda T. A biochemical engineering study of bioproductions using photobioreactor. Seibutsu-Kogaku Kaishi 2008, 86, 117-122. (in Japanese).

30. Terborgh, J.; Ladd, K.V.; McLeod, G.C. Low energy effects of light on growth and pigment content in a yellow-in-the-dark mutant of Chlamydomonas reinhardi. Plant Physiol. 1967, 42, 1665-1672.

31. Alejandre-Durán, E.; Roldán-Arjona, T.; Ariza, R.R.; Ruiz-Rubio, M. The photolyase gene from the plant pathogen Fusarium oxysporum $f$. sp. lycopersici is induced by visible light and alpha-tomatine from tomato plant. Fungal. Genet. Biol. 2003, 40, 159-165.

32. Bayram, O.; Biesemann, C.; Krappmann, S.; Galland, P.; Braus, G.H. More than a repair enzyme: Aspergillus nidulans photolyase-like CryA is a regulator of sexual development. Mol. Biol. Cell 2008, 19, 3254-3262. 
33. Sundstøl Eriksen, G.; Pettersson, H.; Lundh, T. Comparative cytotoxicity of deoxynivalenol, nivalenol, their acetylated derivatives and de-epoxy metabolites. Food Chem. Toxicol. 2004, 42, 619-624.

34. Harris, E.H. A Comprehensive Guide to Biology and Laboratory Use. The Chlamydomonas Sourcebook; Academic Press: San Diego, CA, USA, 1989; Volume 246, pp. 1503-1504.

35. Kam, V.; Moseyko, N.; Nemson, J.; Feldman, L.J. Gravitaxis in Chlamydomonas reinhardtii: Characterization using video microscopy and computer analysis. Int. J. Plant Sci. 1999, 160, 1093-1098.

(C) 2014 by the authors; licensee MDPI, Basel, Switzerland. This article is an open access article distributed under the terms and conditions of the Creative Commons Attribution license (http://creativecommons.org/licenses/by/3.0/). 